\title{
OBRAS HIDRÁULICAS DE ETAPA PREHISPÁNICA EN HUACCANA, CHINCHEROS - APURÍMAC
}

\author{
CiRILO ViVANCO POMACANCHARI \\ INSTITUTO DE INVESTIGACIÓN DE LA FACULTAD DE CIENCIAS SOCIALES \\ UNIVERSIDAD NACIONAL DE SAN CRISTÓBAL DE HUAMANGA \\ civipog@yahoo.es
}

\section{RESUMEN}

En lagunas quchakuna las obras hidráulicas en el Perú prehispánico son admirables, en el largo proceso de las culturas andinas hasta la época Inka el Tawantinsuyu. Antes que arribaran los españoles a los Andes, ningún tipo de infraestructura de contenciones fue implementado en Sudamérica más que en el Perú, las lagunas represadas de cualidad artificial para el riego y consumo humano. El objetivo es detallar las técnicas de construcción, función y componentes de los diques en las lagunas, que son los indicadores y expectativas arqueológicas, organizados en contextos de la instalación y la asociación, los rasgos arquitectónicos y la sedimentología. El resultado del estudio de represas en las lagunas sobre la provisión de agua, relacionadas a las actividades de agricultura y uso doméstico, son obras de ingeniería, dispuestas y construidas con tecnología propia del mundo andino antiguo y con materia prima del lugar. En conclusión, son obras hidráulicas para ampliar, fijar el espejo del agua y desafiar las anomalías naturales en el quehacer cotidiano del hombre en el Perú antiguo.

Palabras claves: Hidráulica, prehispánico, represas, agricultura y riego.

\section{SUMMARY}

In quchakuna waterworks gaps in pre-Hispanic Peru they are admirable, in the long process of Andean cultures until the time the tawantinsuyu Inka. Before the Spaniards arrive to the Andes, any contentions infrastructure was implemented in South America than in Peru, the quality artificial lakes dammed for irrigation and human consumption. The aim is to detail the construction techniques, function and components of the levees in the lagoons, which are the archaeological indicators and expectations, organized in contexts installation and association, architectural features and sedimentology. The result of the study of dams in the gaps on the supply of water, related to farming acti- 
vities and domestic use are engineering works, arranged and constructed with proprietary technology of ancient Andean world and the raw material of the place. In conclusion, they are water works to expand, fix the mirror of water and defy natural anomalies in the daily work of man in ancient Peru.

KeYwords: Hydraulics, prehispanic, dams, agriculture and irrigation.

\section{INTRODUCCIÓN}

Las obras hidráulicas en las lagunas son trabajos de ingeniería, construcciones del mundo andino antiguo en Huaccana Waqana, Chincheros - Apurímac Apwrimaq. El objetivo es entender el conjunto de técnicas sobre las construcciones de estas evidencias del Perú prehispánico, teniendo como pedestal la infraestructura registrada en las lagunas de Tinkiqucha I, Tinkiqucha II y Tuturaqucha o Yanaqucha, ubicadas en la cuenca superior del río Paqchanqa y Pulkay. En el trabajo de campo realizamos en las tres lagunas el registro de obras de contenciones, para evaluar el uso de la materia prima, la tecnología del patrón arquitectónico y determinar el período fundacional de diques, el tipo de compuertas de las mismas que prueban el conocimiento del manejo hidráulico, de actividades agrícolas y de consumo humano, que se concibe la relación indisoluble el hombre-naturaleza con prácticas de cosmovisión, la idea del equilibrio ecológico que contribuye a neutralizar el comportamiento de los fenómenos naturales. Cabe aclarar, para la forma de escribir palabras de quechua se utiliza el sistema propuesto el alfabeto Inka, Urioste (1980).

Se discute la cimentación y la función de las represas, la acumulación y ampliación del espejo de agua, durante la época Inka o el Período Horizonte Tardío (PHT), sin descartar que comiencen dichas prácticas en ocupaciones anteriores. El propósito es identificar las técnicas de las construcciones, el manejo y el control de los diques y las compuertas de obras hidráulicas el conocimiento del equilibrio ecológico, Kendall y Rodríguez (2002 y 2009), Herrera (2012 y 2013) y Regal (2005). En el área de estudio hemos registrado evidencias del embalse artificial para aumentar el volumen almacenamiento de agua en las lagunas naturales, donde construyeron diques con piedras y tierras arcillosas, una obra hidráulica extraordinario, Vivanco (2010 y 2011). Trabajos de ingeniería hidráulica propicias para mejorar las actividades agropecuarias y superar anomalías naturales de la aridez, la desventaja es la alteración del paisaje el deterioro de la formación natural.

El agua es un recurso trascendental para el desarrollo de la civilización, la historia lo ha demostrado con el florecimiento de las culturas, Palerm (1973). En los Andes para aprovechar agua construyeron desde pequeñas estructuras hidráulicas hasta colosales obras, logrando así la distribución equitativa con un conjunto de técnicas andinas sostenibles con el fin de minimizar el impacto ambiental, Treacy (1994), Antúnez de Mayolo (1986) y Ortloff (1985). Es así, al estar basada en la agricultura la economía del Perú prehispánico tuvo una gran inversión en fuerza de trabajo para la construcción de infraestructura, los hombres han dedicado enormes cantidades de energía y recursos para crear las lagunas, reservorios quchakuna y los campos de cultivo en la construcción de andenes patapatakuna y canales yarqakuna. La construcción de qucha requiere de ingeniería hidráulica, el almacenamiento y manejo sensato de agua, Smith et al. (1985), De la Torre y Burga (1989), Denevan (1980), Farrington (1978), Gelles (1986), Parsons y Psuty (1985), Matos (1980), Ravines y Solar La Cruz (1980), Zegarra (1978) y Treacy (1994). Los objetivos logrados en la investigación son el conocimiento de la cuestión hidráulica, las construcciones de diques y sus dependientes, las evidencias arqueológicas en Tinkiqucha 2 y Tuturaqucha así lo confirman.

Las evidencias arqueológicas de las obras hidráulicas registradas es la base de la discusión sobre el manejo de los recursos de agua, que fueron aprovechadas mediante la construcción de represas y 


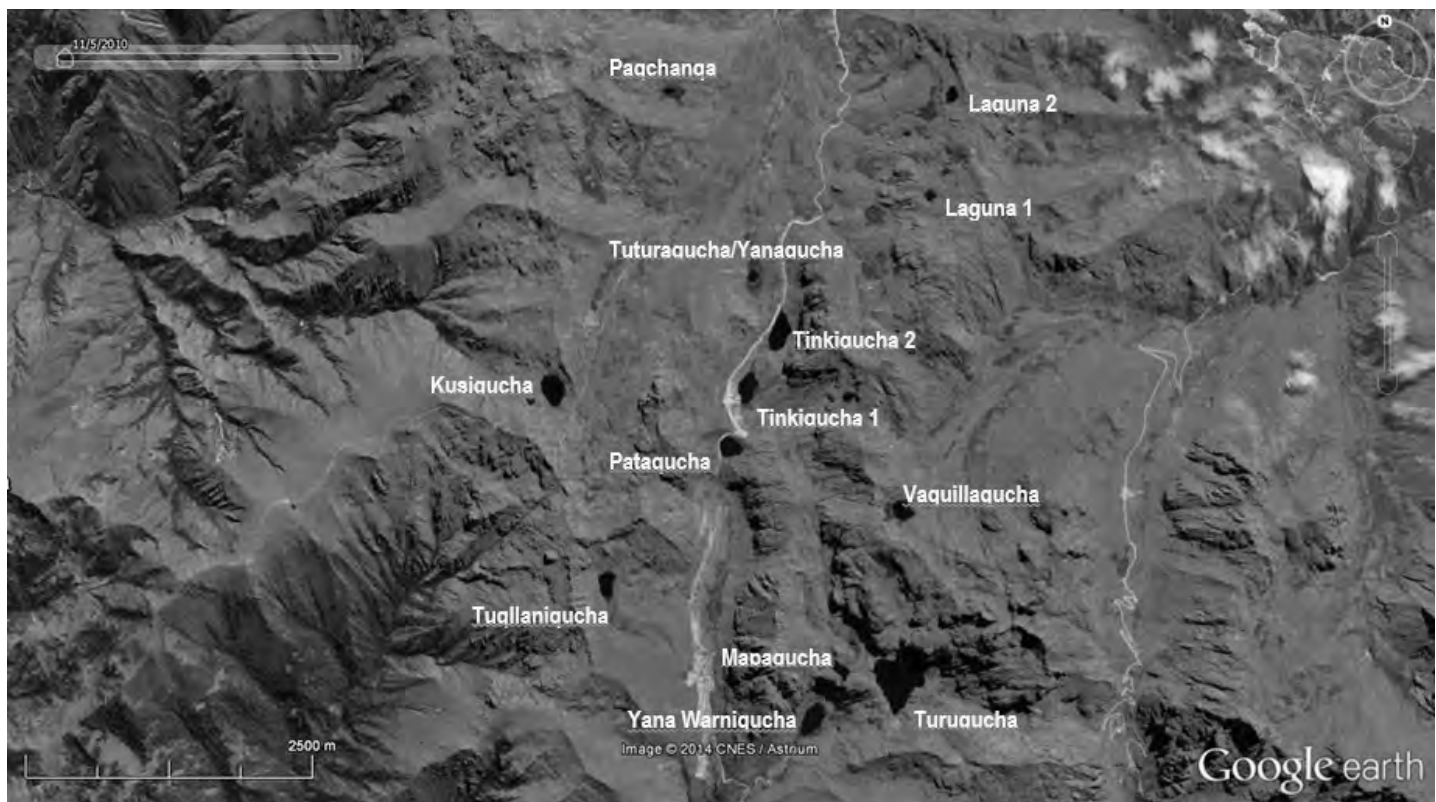

Figura 1: Imagen Satelital. Los trece lagunas registradas, que forma parte del proyecto obras hidráulicas quchakuna de la época prehispánica en Huaccana, Chincheros - Apurímac. Véase la distribución en el paisaje y las quebradas

las bocatomas en las lagunas naturales. El objetivo de la construcción de diques es para aumentar el volumen y espejo de agua en tiempo de lluvias y su utilización en meses secanos en diferentes actividades. Es una construcción de ingeniería hidráulica una tecnología propia para controlar y distribuir el elemento vital del consumo humano y el de riego parquy de actividades agrícolas. En base a las evidencias planteamos la hipótesis, que las tierras de las quebradas de Paqchanqa y Pulkay requerían las aguas de las tres lagunas, las construcciones de represas y sus bocatomas del manejo y control de agua, responde a esta necesidad. El agua es el elemento principal para las actividades agrícolas y consumo humano, estas han sido aprovechas mediante la infraestructura hidráulica de obras como los reservorios más pequeños, construcciones de canales primarios y secundarios que regulaba el uso adecuado.

\section{Examen General de Obras Hidráulicas}

El estado de la cuestión la obra hidráulica en el Perú antiguo es interesante, sin embargo existen muy pocos estudios al respecto. Arqueológicamente el área de estudio es desconocida a falta de trabajos de investigación, el tema aún no ha sido abordado por descuido de los investigadores interesados en el cambio climático, nuestro interés es entender como los antiguos hombres resolvieron anomalías naturales: la escasez de agua. Las obras hidráulicas del Perú prehispánico detenidos por la administración española en el virreinato, se conocen a partir de estudios de historia, etnohistoria, etnografía y arqueología, Kendall (1992), Letchman y Soldi (1985), Morales y Masson (2002); las evidencias arqueológicas son canales, reservorios y elementos dependientes.

Las lagunas en Apurímac, son de origen glaciar, dispuesto por cadenas de montañas extendidas de sur a norte, resaltan Pacucha, Antacocha, Suitococha, Matará, Huacullo, Occocco y otros. La orografía de Apurímac cruza en direcciones diferentes, definido por la sucesión de montañas, que la configuran y le dan la apariencia "papel arrugado" que señala A. Raimondi. El área de estudio se ubica a 9 km del 
distrito de Huaccana, altiplanicie de la región puna, al pie la cadena elevada el apu 'Ayavi', el cerro más imponente de la zona que constituye un ramal de la cordillera Vilcabamba, CRDCA (2004). Una cadena de lagunas Tinkiqucha 1, Tinkiqucha 2 y Tuturaqucha/Yanaqucha, el agua de las tres se conectan y alimentan al río Paqchanqa, luego a Pulkay y Pampas.

Los antiguos peruanos dominaron el comportamiento del clima con intuición y conocimiento del territorio y el entorno, a los recursos naturales manejaron equitativamente, asumiendo la estrecha relación hombre-naturaleza. En obras hidráulicas invirtieron el enorme esfuerzo para racionalizar la escasez de agua, construyendo reservorios en las lagunas. Los estudios de infraestructura agrícola rara vez se refieren a obras de ingeniería y elementos relacionados de almacenamiento y conducción de agua. Al respecto existen descripciones de los siglos XVI y XVII y estudios recientes de historiadores y arqueólogos, el registro en la zona sur de Ayacucho, Apurímac y Cusco, con plan de rescatar la tecnología andina, Kendall y Rodríguez (2009). La construcción de quchakuna el represamiento de agua requiere el conocimiento de ingeniería hidráulica, la elección de lugar indispensable para fundar la arquitectura con piedras y tierra arcillosa de tipo impermeable. Los datos tomados por los españoles de obras hidráulicas en la costa y sierra fueron los andenes, canales y represas, Rabey (1987). Cimentaron represas aprovechando la formación natural de las lagunas para fines agrícolas y consumo humano, los vestigios de represa y la compuerta de agua aún se observa en Antacocha en las nacientes del río Santa Eulalia y otras en la cordillera Negra, Gelles (1986) y Lane (2013).

Los antiguos peruanos construyeron imponentes obras de ingeniería hidráulica, desde las formas tempranas de este tipo de infraestructura hasta el proyecto ambicioso de los Inka, interrumpido por el nuevo sistema de administración virreinal, Denevan (1980) y Farrington (1978). El talento científico y genial de nuestros antepasados, son la geografía, ingeniería civil, hidráulica, economía, agronomía; los acueductos de Nazca, el canal Cumbemayo de Cajamarca, los andenes de Moray de Cusco y entre otros, Earls y Silverblatt (1985), González (1978). Estas obras hidráulicas aún visibles en la sierra centro sur, son el canal de Wari en Ayacucho, Tipón, Pikillaqta y otros en Cusco, Huancané y Laymecocha-Soras en Ayacucho y otros en los Andes centrales; que comprenden represas, acueductos, canales de conducción y vertederos, Alegría (2008), Kendall y Rodríguez (2009). Los españoles a su llegada al Perú, describieron de increíble la organización de los antiguos peruanos que tuvieron una satisfacción efectiva de las demandas de agua en sus quehaceres cotidianas.

La etnohistoria revela que el Tawantinsuyu estaba asociado a grandes trasformaciones sociales y políticas que surgieron a causa de sequías y otros trastornos naturales. La alta frecuencia de sequías, inundaciones, plagas, pestes y heladas; condicionaron la evolución social, política y la tecnología. La exagerada inestabilidad y la extrema heterogeneidad del sistema ambiental andino exigieron la formación de organizaciones para lograr un desarrollo eficiente, Earls y Silverblatt (1985). Estas habilidades indican un manejo de principios de la cibernética, la teoría de sistemas y la decisión para manejar la incertidumbre temporal y la alta heterogeneidad espacial, que permite deducir la existencia de la racionalidad ecológica el uso sostenible de la biodiversidad, Earls (2008).

Los Andes centrales fueron afectados por eventos climáticos, anomalías naturales de la madre naturaleza desde tiempos ancestrales, el arribo de los primeros grupos humanos hasta la época Inka, el fenómeno El Niño, sequias y heladas. A estos fenómenos climáticos nuestros abuelos se enfrentaron, con una visión mística de los astros, la veneración a los recursos agua y tierra, sobre todo respeto a la naturaleza. El adecuado uso de agua en el Perú antiguo fue la base de sociedades agricultores, concibiendo el desarrollo tecnológico de la producción para alimentar numerosas poblaciones, Gonzales y Trivelli (1999), Soldi (1982). Es necesario recalcar que la disponibilidad de agua es irregular en la zona andina tanto en el tiempo y en el espacio. De ahí, el uso de represas para retener agua y los sedimentos 
que tiende a complementar la modificación de superficies de cultivo en terrazas y sistema integral agro pastoril. Ante la irregularidad hídrica reciente, los reservorios antiguos merecen la mayor atención de especialistas, Herrera (2012), Denevan (1980) y Lane (2007).

Las represas filtrantes de la cordillera Negra, no fueron diseñadas para la agricultura, es para el pastoreo. Tal vez por ello, escapan de la atención de arqueólogos. Existen represas estacionales antiguos que siguen siendo utilizadas y mantenidas por pueblos andinos asentadas en lugares áridos alto andinas. La ausencia de represas artificiales a lo largo de la cordillera central de los Andes centrales se vincula a la presencia de abundantes glaciares, el deshielo ofrecen fuentes de agua estables que hacen menos urgente la construcción de represas, Herrera (2012). Sin embargo, los antiguos peruanos aprovecharon las lagunas naturales, acondicionando en ella las obras hidráulicas de control y manejo del agua yaku yachachiy. La mayoría de las lagunas por no decir todo, en la sierra han sido alteradas con infraestructura hidráulica. La cronología de las represas es difícil determinar con precisión a partir de evidencias disponibles, Denevan (1980). La mayoría de represas parece que datan de los últimos siglos antes del arribo de los españoles, aunque no se descarta diseños anteriores del Perú prehispánico.

\section{Acumulación y Elaboración de Datos Empíricos}

La elección del área de estudio es resultado de una evaluación acerca las características de obras hidráulicas de almacenamiento, control y uso de aguas de las lagunas. El trabajo de campo interdisciplinario: arqueológico, histórico y geográfico involucra un estudio integral y a la vez labores planificados, el uso de materiales equipos e instrumentos apropiados de prospección arqueológica y heurística, con participación de profesionales de mucha experiencia.

El tipo de investigación es básica, el nivel de examen es la observación y analítico sobre el estado de la cuestión de las represas y sus componentes. Los métodos empleados son: descriptivo, deductivo e inductivo y comparativo. El diseño de investigación es por objetivo y las técnicas e instrumentos concernientes del trabajo de campo y gabinete son de especialidades de arqueología, historia y geografía.

Metodológicamente, los datos empíricos se recolectaron en tres etapas. Primero se elaboró los croquis de la infraestructura existente de ingeniería hidráulica con apoyo de GPS navegador, complementado el registro y la descripción de las evidencias arqueológicas y la biodiversidad. Segundo se evaluó la cimentación y la función de diques y sus componentes de las lagunas, así como el control, manejo y el uso de aguas. Tercero se hizo el intento de explicar la cuestión hidráulico la relación entre la infraestructura de diques, de agricultura y de consumo humano.

La intervención del trabajo de campo se hizo con equipos e instrumentos según la metodología y técnica planificada utilizando diversas fichas de registro. Las lagunas con evidencias de represas se ubicaron con GPS navegador mediante las coordenadas UTM (datúm WGS 84) para incorporar un punto en el mapa como sitios arqueológicos. El lineamiento metodológico y técnico del proyecto, es la siguiente:

Primero: ubicación precisa de cada sitio lagunas con represa, con GPS navegador y descripción en detalle una observación directa in situ.

Segundo: registro arquitectónico diques de cada sitio lagunas con represas para determinar la cimentación y la funcionalidad de obras hidráulicas, en libretas, fichas, gráficos y fotos.

Tercero: se elaboró croquis de elementos arquitectónicos visibles de diques y sus componentes de reserva y control la bocatoma de las lagunas. 


\section{Las Obras Hidráulicas de Huaccana}

Se presenta los resultados del trabajo de campo y gabinete, una evaluación interdisciplinaria con enfoque de geografía, arqueología e historia. De observación directo in situ las características geográficas y las evidencias arqueológicas, descripción en detalle del paisaje natural y cultural, se incluye el peritaje de archivos de los distritos de Huaccana y Ongoy Unquy, Atlas del Perú (2009). Destaca en los datos arqueológicos: la presencia de los restos de represas en las salidas de agua de las lagunas, la cimentación y las técnicas de construcciones de diques y sus componentes, obras hidráulicas de reserva para la agricultura y posible consumo humano. Registramos un total de trece lagunas con evidencias de construcciones de las obras hidráulicas, cimentaciones de diques sin presencia de material cultural mueble: cerámica, líticos y otros artefactos, que auxilie precisar la cronología; el trabajo sistemático sólo se hizo en lagunas.

La descripción geográfica del departamento de Apurímac, reportan los primeros cronistas que describieron como la tierra muy doblada y poderosamente fangosa, por la formación natural accidentada. El área de estudio se ubica en la cadena central de la cordillera de los Andes, dispuesta por las altas montañas los cerros de Yanawarmi que significa mujer negra, cresta que corre de este a oeste del pico imponente el apu Ayavi, la deidad regional en la zona noroeste de Apurímac. En concavidades y debajo de estas vertientes inclinadas, agrestes y encrespadas cumbres y otros vértices más pequeños formaron las lagunas alto andinas de origen glaciar. Estas lagunas ocupan las depresiones que en otros tiempos fueron los fondos de los circos glaciares llamados anfiteatros, hondonadas de acumulación de nieves y hielo, debajo están los pequeños valles alto andinos con presencia de morrenas, evidencias de erosiones creadas por glaciares, más abajo se muestran los valles o quebradas en forma de $\mathrm{U}$ y V.

Las lagunas presentan poca profundidad y muestran un talud escasamente pronunciado en el fondo, razón por lo que en sus orillas se observa plantas de totora y otras gramíneas. Las lagunas Yana Warmiqucha, Pataqucha, Tuqllaniqucha, Turuqucha y Mapaqucha, se ubican en la vertiente lado sur del cerro apw Ayavi; mientras las lagunas Tinkiqucha 1 y 2, Yanaqucha, Wakillaqucha y otros; se encuentra en la vertiente norte. Las orillas fueron rellenadas de material por la erosión de las laderas pendientes, que reduce su verdadera dimensión de las lagunas. Todas estas lagunas tienen la entrada y salida de aguas, la formación natural fue alterada con la cimentación de diques.

La laguna Tinkiqucha 1 es el espejo de agua de forma alargada y amorfa, el fondo de talud tiene poco pronunciado. Las aguas que fluyen de esta laguna alimentan a Tinkiqucha 2, esta se ubica en la parte inferior a unos $300 \mathrm{ml}$. siguiendo el curso del agua. La salida del agua es al lado norte, en la orilla de lado derecho se observa la intervención humana, la cimentación de un muro de dos hiladas para la construcción de obra hidráulica dique, el vivo testigo a pesar del tiempo transcurrido, mide 2.60 a 2.85 $\mathrm{m}$ de ancho y $10 \mathrm{~m}$ de largo, piedras colocadas en dos hiladas que explica el inicio de la obra; en el lado izquierdo a la misma altura de estos restos existe un pequeño promontorio de afloramiento rocoso, al parecer tenían planificado conectar la construcción en proceso a esta roca.

La laguna Tinkiqucha 2 es alargada y se alimenta de aguas que afluyen de Tinkiqucha 1, se ubican en la misma depresión la diferencia de altitud es $7 \mathrm{~m}$. Toponimia Tinkiqucha en quechua runa simi significa dos lagunas yuxtapuestas. La bocatoma tiene salida natural por donde fluyen aguas que alimentan más abajo a unos $230 \mathrm{~m}$ siguiendo el itinerario del riachuelo a Tuturaqucha o Yanaqucha. En su lado norte y lugar donde se estrecha la forma de la laguna, se observa un muro de dos hiladas con aparejo de guijarros y tierra arcillosa de color negruzco y rojizo, mezcla que da dureza firme, a fin de evitar el desgaste por efecto de lluvias. La evidencia arqueológica destaca la construcción de la represa que mide $17 \mathrm{~m}$ de largo, entre 2.50 y $2.80 \mathrm{~m}$ de ancho y una altura de $1.20 \mathrm{~m}$., para almacenar, 


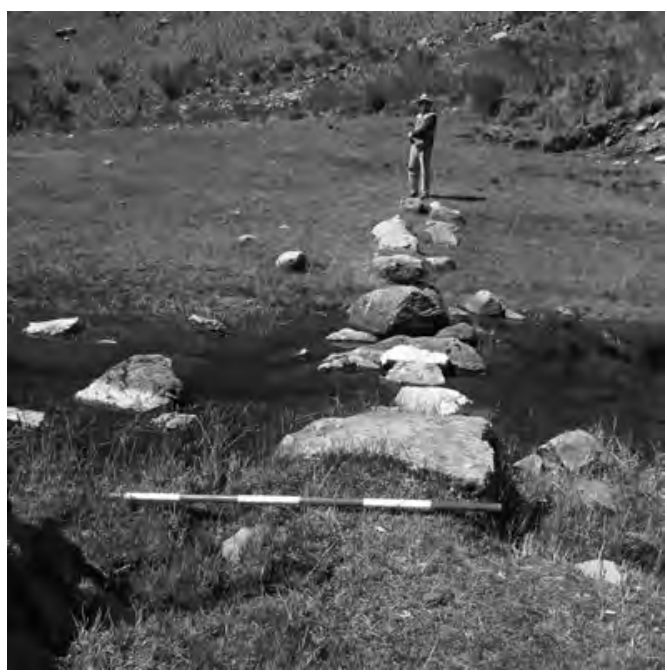

Figura 2: laguna de Yana Warmiqucha, véase restos del muro de dique en la salida de agua, evidencias arqueológicas que testifican las obras hidráulicas.

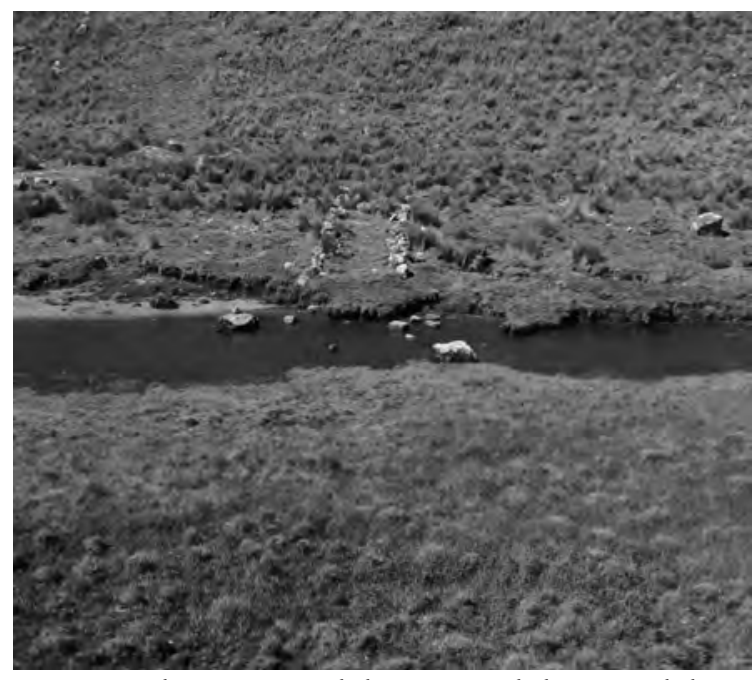

Figura 3: la cimentación de la represa en la bocatoma de la laguna de Tinkiqucha 1, véase el ancho y la orientación, al parecer no terminaron construir, tal se interrumpe con la llegada de los españoles.

controlar y manejar las aguas. Ambas caras del muro presentan un paramento irregular, construidas con piedras seleccionadas del lugar y esta devastada el centro de la represa; en las inmediaciones hay piedras apiladas dispuestas a ser utilizadas, que indica la paralización intempestiva de esta obra hidráulica.

La laguna Tuturaqucha o Yanaqucha, se ubica en la misma cuenca donde están Tinkiqucha 1 y Tinkiqucha 2. Toponimia Tuturaqucha significa laguna de totora, mientras Yanaqucha expresa laguna negra. Presenta poca profundidad el fondo de la laguna, se observa al interior de la misma abundante cantidad plantas de totora y otras, de mucha utilidad para la fauna volátil silvestre. En la salida del agua se observa la monumental obra hidráulica, muro doble de dos hiladas ambas adosadas con precisión, la más alta y larga al sur y la más corta y baja al norte. El área de desaguadero está alterada, solo se observa la concentración de piedras de diferentes tamaños entre ellas talladas que forman parte de obras de ingeniería de control y manejo. El muro 1 es el más corto tiene $23 \mathrm{~m}$ de largo y $2.50 \mathrm{~m}$ de ancho, mientras el muro 2 tiene un largo de $35 \mathrm{~m}$ y un ancho de 2.30 a $2.50 \mathrm{~m}$ y presenta la parte más alta de $1.60 \mathrm{~m}$ de altura.

El aparejo de los dos muros paralelos presenta disposición y trabazón de materiales empleados, de textura compacta de guijarros y tierra arcilla de color oscuro, de carácter impermeable. Los muros de contención se construyeron con grandes bloques de piedra alargada y amorfa, entre ellas algunas talladas de forma rectangular; el sumidero posible fue una abertura estrecha posible circular, preparadas con grandes y largas rocas labradas extendidas en el piso para evitar el desgaste del suelo que podría afectar la base de la represa. Cerca el dique en lado izquierdo aún se mantiene el depósito de hormigón material no utilizado, también en ambos lados del muro de contención se observa el apilamiento de piedras para la utilización, el muro del lado norte parece ser que estaba en plena construcción como una estructura de refuerzo o contención, para asegurar la seguridad de la obra de ingeniería, restos que en la actualidad están abandonadas, destruidas y desmanteladas.

Las lagunas descritas presentan una formación natural para la acumulación de agua, que fueron aprovechadas para estirar el espejo con construcciones de obras de ingeniería monumental. Se ubi- 

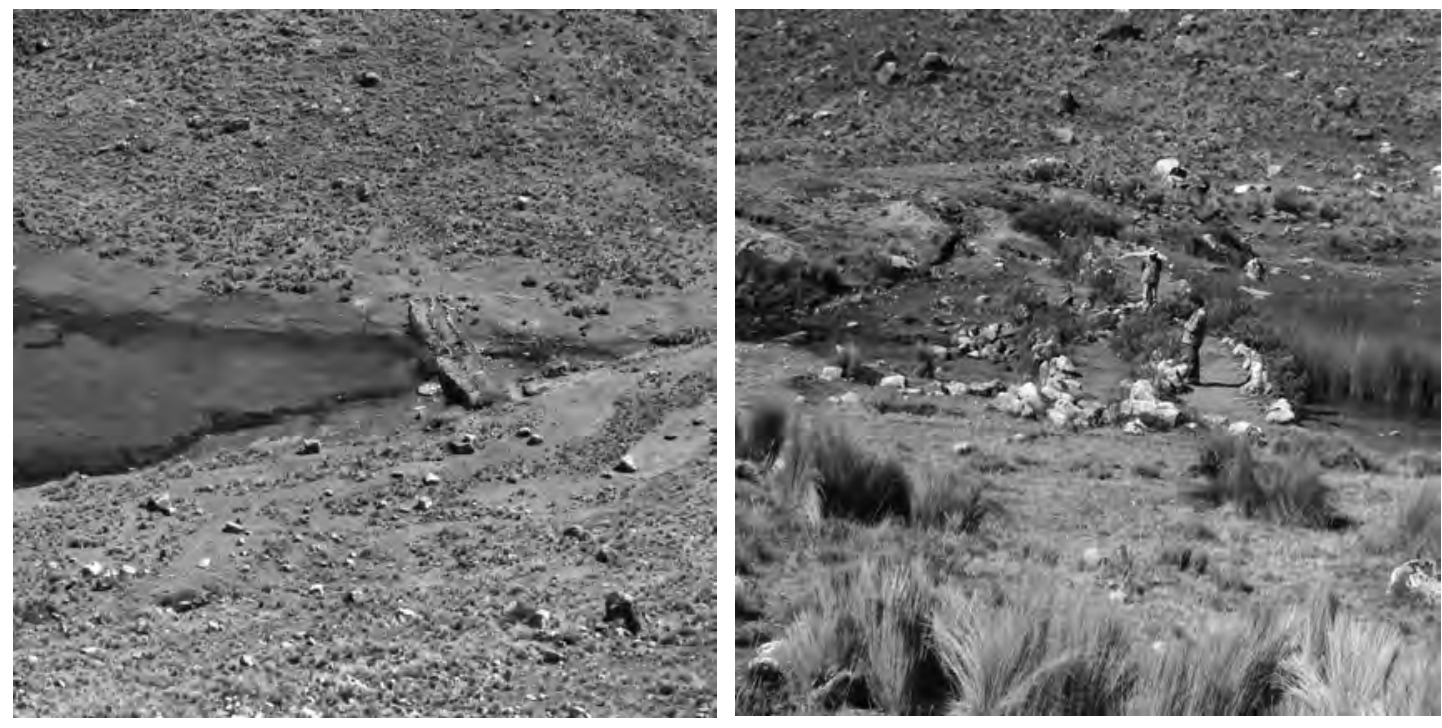

Figuras 4 y 5: muro doble adosada de la represa de Tuturaqucha/Yanaqucha, véase los restos que atestiguan la importancia de la obra de ingeniería durante la época Inka.

can en la región puna, territorio eriazo y zona de pastoreo comunal, al sur y parte alta de las quebradas Pulkay y Paqchanqa, Vivanco (2010 y 2011 Ms). También cerca y al costado de estas lagunas cruza la red vial antiguo, y a la vez se ubica al pie de la cadena de montañas que rodea a la cubeta, una depresión que muestra haber sido antigua depósito de masa de hielo y después del deshielo se quedó convertido en el depósito de agua. Las tres lagunas en cadena están rodeadas de montañas a los costados, lados este, sur y oeste, dejando abierta en el lado norte una explanada de dos niveles, posible caída, lugar por donde fluye el riachuelo que le conocen con este nombre Paqchanqa y más abajo toma el nombre de río Pulkay.

Las otras lagunas inspeccionadas son de diferentes tamaños que presentan las evidencias de intervención humana. Pataqucha, se halla ubicada exactamente en la abra el inicio de dos vertientes: las quebradas Paqchanqa y Tuqllani, el desagüe se orienta hacia el sur oeste donde se percibe algunos bloques de piedras canteadas esparcidas que revela la intervención humana. La laguna Yana Warmiqucha, se ubica en una depresión (la cubeta o circo glaciar), de forma alargada y la abertura estrecha está excavada, en el fondo de dos laderas abruptas, para abrirse el paso longitudinal cuesta abajo, en este lugar se observa la construcción de obra hidráulica con bloques de piedra canteadas, colocadas de largo en una seriación lineal la cimentación de dos hiladas. La laguna Azulqucha o Turuqucha, en su desaguadero se observa concentración de piedras de tamaños diferentes que explica la alteración. Mapaqucha se ubica en la parte superior de Yana Warmiqucha y al oeste de Turuqucha. Wakillaqucha se ubica en la parte más elevada de la cadena de montañas que corre de este a oeste, es decir, del pico Ayavi al lado oeste, se observa la concentración de piedras que parecen formar el dique, una alteración intencional. Tojillan o Tuqllaniqucha, toponimia tuqllani significa trampa para atrapar perdiz yutu y qucha laguna. En la bocatoma se observa piedras dispersas que forma parte de la cimentación del muro.

La laguna Cusicocha Kusiqucha nombre designado en Carta Nacional del Perú, los lugareños conocen con el nombre Maqmaqucha. La bocatoma se ubica en su lado noroeste, donde se aprecia el apilamiento de piedras, parte del muro de contención dique. Existen otras tres lagunas en ambos lados de la sub cuenca superior de Paqchanqa, a uno de ellos estamos denominando con este mis- 


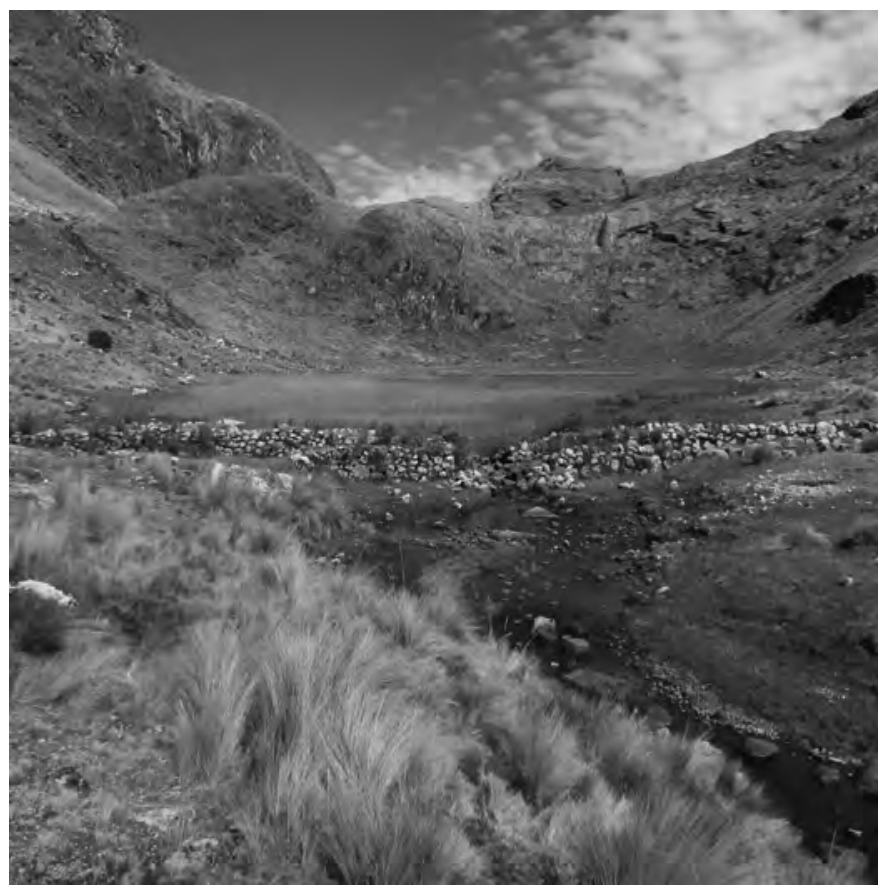

Figura 6: laguna de Tuturaqucha/Yanaqucha, véase el dique muro doble adosada en la salida de agua. La obra hidráulica monumental de la época Inka. mo nombre, mientras a los dos restantes con el nombre la laguna 1 y 2 . Los tres presentan la modificación del hombre en la bocatoma que explica la represa artificial con el fin de extender el espejo de agua. En ninguna de las lagunas registradas como sitios arqueológicos por la cimentación de diques hemos encontrado evidencias de la cultura material, como cerámica y otros elementos culturales muebles. La alteración a la forma natural de las lagunas, son la presencia de construcciones diques muros de dos hiladas en las bocatomas para ampliar el espejo de agua, orientadas a la actividad agrícola y consumo humano, transportadas mediante construcciones de obras hidráulicas.

Asociado a obras hidráulicas en las lagunas Tinkiqucha 1 y 2, Yanaqucha o Tuturaqucha, existen otros elementos relacionados a la conducción de agua. En inmediaciones de Tuturaqucha existen evidencias de viviendas con corrales que podrían ser la residencia de personas que vigilan, controlan y mantenían el dique doble. Aún no hemos ubicado las bocatomas de canales en ambos lados de la quebrada, posible existen este tipo de infraestructura hidráulica de riego en la parte inferior, también los reservorios quchakuna más pequeños, canales primarios y secundarios para el riego en andenes y el consumo doméstico. Al respecto la información oral es suficiente, los lugareños nos cuentan de fragmentos de canales y reservorios antiguos abandonados en los extremos o cerca de sus chacras, en la parte baja de la quebrada, hoy en día son tierras agrícolas abandonadas, sin embargo a simple vista se observa la andenería que demuestra la complejidad.

\begin{tabular}{|l|l|l|l|l|l|l|}
\hline Nombre & Este & Norte & Altit. & \multicolumn{1}{|c|}{ Evidencias } & Dimensión & \multicolumn{1}{c|}{ Otras elementos } \\
\hline Yana Warmiqucha & 637849 & 8510175 & 3655 & Muro de represa & $308 \times 158 \mathrm{~m}$ & Entrada y salida de agua \\
\hline Pataqucha & 637443 & 8512438 & 4152 & Concent. piedras & $183 \times 173 \mathrm{~m}$ & Entrada y salida de agua \\
\hline Tinkiqucha 1 & 637751 & 8513229 & 4074 & Muro inicio & $303 \times 155 \mathrm{~m}$ & Entrada y salida de agua \\
\hline Tinkiqucha 2 & 638002 & 8513636 & 4067 & Muro de represa & $355 \times 155 \mathrm{~m}$ & Entrada y salida de agua \\
\hline Yanaqucha & 637806 & 8514146 & 3980 & Doble represa & $392 \times 148 \mathrm{~m}$ & Entrada y salida de agua \\
\hline Turuqucha & 638587 & 8510489 & 4083 & Muro de represa & $447 \times 427 \mathrm{~m}$ & Entrada y salida de agua \\
\hline Mapaqucha & 638286 & 8510599 & 4030 & Concent. Piedras & $164 \times 62 \mathrm{~m}$ & Entrada y salida de agua \\
\hline Wakillaqucha & 638957 & 8511914 & 4249 & Concent. Piedras & $190 \times 178 \mathrm{~m}$ & Entrada y salida de agua \\
\hline Kusiqucha & 636027 & 8513206 & 4161 & Muro de represa & $278 \times 210 \mathrm{~m}$ & Entrada y salida de agua \\
\hline Tuqllaniqucha & 636464 & 8511350 & 4198 & Muro de represa & $252 \times 175 \mathrm{~m}$ & Entrada y salida de agua \\
\hline Paqchanqa & 637303 & 8515440 & 4077 & Muro de represa & $172 \times 103 \mathrm{~m}$ & Entrada y salida de agua \\
\hline Laguna 1 & 639279 & 8514487 & 4194 & Muro de represa & $103 \times 101 \mathrm{~m}$ & Entrada y salida de agua \\
\hline Laguna 2 & 639531 & 8515255 & 4148 & Muro de represa & $125 \times 90 \mathrm{~m}$ & Entrada y salida de agua \\
\hline
\end{tabular}

Tabla $N^{\circ} 01$. Elaboración propia, lagunas y evidencias de obras hidráulicas. 
La importancia de andenes y terrazas agrícolas en la quebrada Paqchanqa y la micro cuenca de Pulkay asociada a las obras hidráulicas. La población andina aún mantiene en porcentaje mínimo la experiencia de siembra en andenes y terrazas, sin embargo los problemas ambientales -el cambio climático, el deterioro de los recursos naturales, la contaminación de suelos y aguas, la reducción de la biodiversidad- son impactos que contribuyen el abandono sistemático de prácticas agrícolas ancestrales, además no es rentable por fuerte inversión de fuerza de trabajo. Los andenes evitan la erosión de suelos, ayudan a regular los flujos de agua, mantienen la humedad y forman parte de una agricultura que contribuye al mantenimiento de la biodiversidad, a embellecer el paisaje, a utilizar las energías renovables y no contaminantes, de modo que, la práctica agrícola en andenes es sostenible en relación a la naturaleza, la misma que contribuye a desafiar los impactos negativos del cambio climático del pasado y presente.

\section{Yaku Mama: Sistemas Hidráulicos y Alteraciones Naturales}

La madre agua yaku mama en la cosmovisión andina es el concepto de la 'crianza' el recurso agua el elemento principal de todos los seres vivos. Hay una correspondencia entre la lengua y el lugar donde se encuentra, de término general es la madre agua yaku mama, el lago y las lagunas mama qucha que se diferencia del mar hatun mama qucha y lagunas uchuy mama quchakuna, además los ojos de agua se define pukiwpi mama yaku, el río mayu yaku mama, agua del canal yarqa yaku mama y de la lluvia para yaku mama. En la epistemología andina, la forma de conocer, pensar, hablar y hacer es sintiendo que todo lo que existe son seres vivos y por lo tanto son padres y madres, y tienen el espíritu; de ahí la construcción sobrenatural de los dioses tutelares apwkuna y wamanikuna, Mendoza y Campos (s/f) y Cáceres (2002). De modo que, el agua yaku mama es madre, porque es sangre de la madre tierra pacha mama, su conservación es ritual donde participa todo el pueblo, razón por lo que erigieron sistemas hidráulicos con la intención de vigilar las alteraciones naturales.

Las lagunas en quechua runa simi se escribe de distinta manera: cocha, qocha, cochas, ccochas, gochas, qochas, qucha, quchakuna, "ccocha", González (1989 [1608]). Se trata de lagunas naturales alteradas con obras hidráulicas, que en el mundo andino antiguo jugó un papel fundamental, como componente de la actividad agropecuaria y consumo humano. En la sierra centro sur del Perú las lagunas naturales de diferentes dimensiones fueron modificados por el hombre, con obras de ingeniería para almacenar en tiempo de lluvias puquy killakuna; en sus desaguaderos construyeron diques y sumideros para ensanchar y controlar el espejo de agua. Los reservorios artificiales con uso temporal de dimensiones pequeñas se construyeron en la región suni y quechua para acumular poca cantidad de agua, estaban directamente asociadas a las parcelas con riego y muchos aún están en uso.

Las obras hidráulicas, los diques y los canales del Perú prehispánico es el desarrollo tecnológico de almacenamiento, control y manejo sistemático de agua. Estas construcciones de represas forman parte de prevención frente a la escasez de agua perturbada por el régimen pluvial anómalo, ejemplo sequía o mucha lluvia que deja estragos en la sociedad andina. El tiempo de lluvias estacionales fueron aprovechados de manera sistemática para aumentar el volumen de agua en las lagunas naturales, para ello construyeron diques, a esta actividad denominaron la cosecha de agua yaku quñuy, el dinamismo que desarrollaron las construcciones, así como el mantenimiento o limpieza con fiestas, grandes celebraciones de banquetes mikuy y bebida chicha aqa, ejemplo el caso limpieza del canal yarqa aspiy. Los restos de infraestructura hidráulica constituyen parte de una actividad social, para remediar la sequía, en otras palabras estas evidencias se manifiesta ¿Cómo enfrentar el impacto socio ambiental provocado por el cambio climático? fenómenos climatológicos, que generan riesgos por exceso o escasez de agua. 


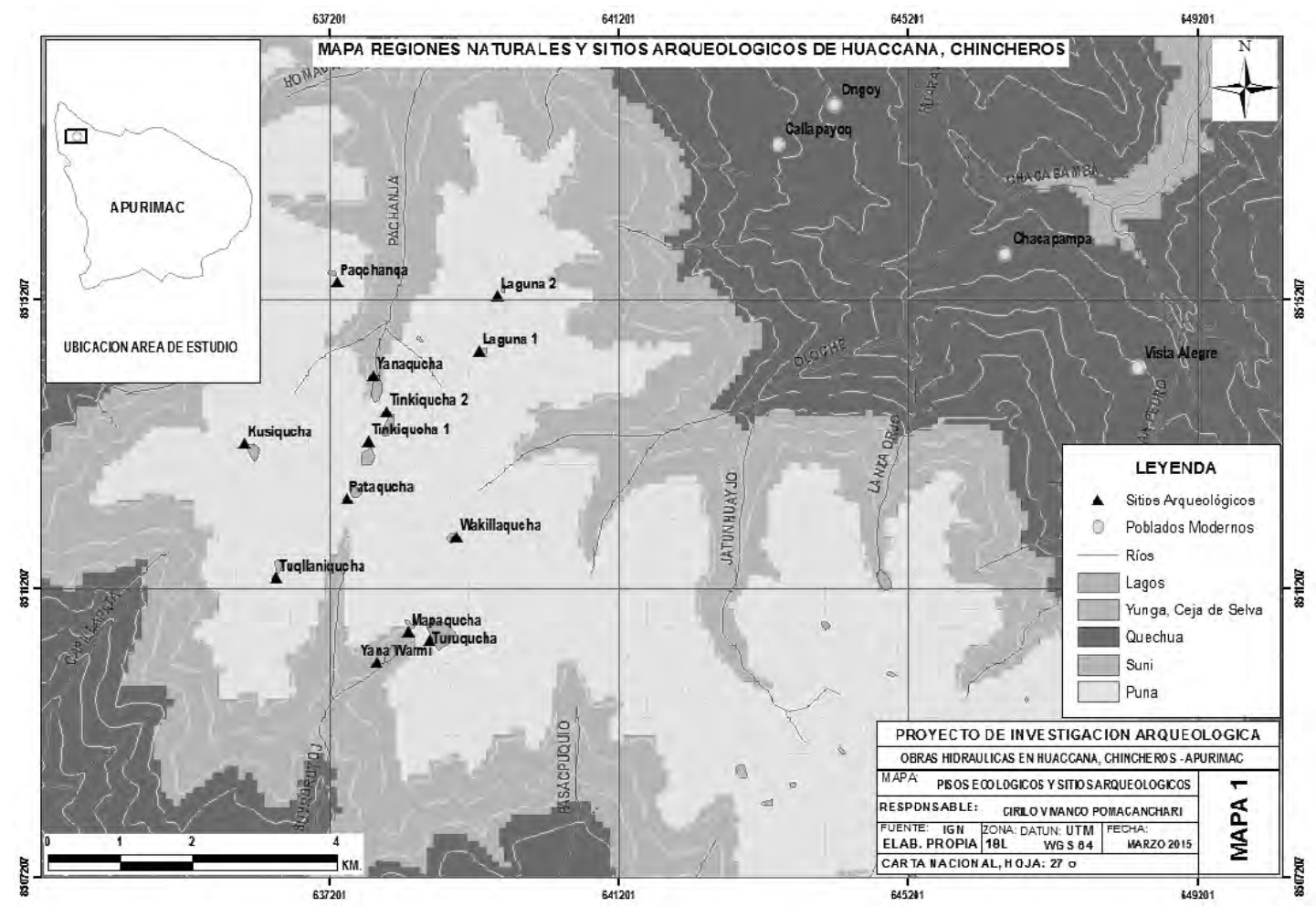

La agricultura prehispánica estaba marcada de cosmovisión por la estrecha relación el hombre, territorio y su entorno. De manera que, se desarrolló una ciencia basada en supersticiones, que producen las explicaciones fantásticas sobre los fenómenos naturales e incluso para orientar el discurso a fines políticos y religiosos. Señalan que los arqueólogos, descubrieron las alteraciones climáticas y una enérgica práctica de la cosmovisión en el Perú prehispánico, Huertas (2001, 2003 y 2006), Carrión (2005 [1955]) y Zegarra (2011). En el diccionario de González Holguín, hemos encontramos más términos quechuas que refieren a fenómenos naturales, que demuestran las alteraciones del modo de vida en el Perú antiguo, ejemplo heladas ccaça ccaça qasan, granizadas runtun, terremotos pacha kuyun, inundaciones lluqlla o tiwsqan, anomalías naturales que ocasionaban los estragos a la sociedad andina antigua, González (1989 [1608]).

Entonces, hay evidencias que demuestran que en la etapa prehispánica, el fenómeno del Niño como otros eventos atmosféricos estaba relacionado a causas divinas. Las variaciones climáticas eran percibidas como castigo de dioses por lo que la sociedad debía realizar sacrificios humanos para calmar su ira y clamar el retorno a la normalidad. El fenómeno del Niño con lluvias muy fuertes es considerado en la cosmovisión andina como pachacuti del agua; Rostworoski (2001) y Huertas (2001), revelan las alteraciones naturales que influyen de manera directa y profunda en la religiosidad del hombre andino, estas son recurrentes y durante milenios impactaron a las poblaciones creando problemas, como el caso de la costa central, Ávila (1966 [1598]). El poblador andino frente a las calamidades necesitaba el apoyo colectivo y de la protección de sus deidades, situación que implicó un comportamiento recíproco y contraprestación a la naturaleza. En este contexto los sacerdotes que servían de nexo entre el hombre y sus deidades, tuvo que observar con regularidad el tiempo y los ciclos productivos para ofrendar a los dioses andinos. 
El Fenómeno del Niño en proporción a mega Niño, influye en la religión andina buscando mecanismos de protección en sus dioses contra la acción devastadora. Se deduce la creación/ representación de dioses protectores con poderes capaces de calmar lluvias torrenciales. El grupo sacerdotal, en su rol de nexo entre el hombre y las divinidades, conocen y predicen la alteración natural, la existencia de canónigos actualmente llamadas meteorológicas, por ejemplo ofrendas a lagunas quchankuna, Pardo (2009) y Berrocal (2014). Esto confirma la hipótesis sobre la existencia de grupos conocedores en el tema del drenaje de agua, de la filtración y humedad del terreno, obras hidráulicas de almacenamiento y de conducción, Lane (2007 y 2013), las obras de ingeniería en la cordillera Negra en Ancash.

Esta especialización es más notable en la época Inka, la reforma de la administración del tiempo y la ecología con obras hidráulicas monumentales. Frente a las devastaciones de fenómenos naturales que agobiaban la vida cotidiana, exigían la información rápida y precisa que lograban los sacerdotes que eran filósofos y astrólogos, Guaman Poma de Ayala (1936 [1615]), eximios cateadores de la naturaleza. Todo lo referido con la creación de términos, influencia en la religión, especialización, junto a otras reacciones, se identificó culturas que se desarrollaron en el espacio geográfico del distrito de Huaccana, evidencias arqueológicas que demuestran el conocimiento, sobre anomalías naturales y la distribución equitativa del agua en la cuenca de Paqchanqa.

La presencia de eventos extremos en el Perú prehispánico, como lo son las inundaciones y las sequías, son anomalías naturales por el cambio climático. Las catástrofes provocadas por riadas en algunas zonas, así como sequías prolongadas en algunas partes de los Andes centrales, provoca pérdidas económicas. El riesgo sería causado por las lluvias o altas precipitaciones, que podrán aumentar la erosión del suelo y producir la sedimentación de reservorios y ríos, afectando la infraestructura en algunos casos. En cambio, la ausencia de lluvia normal y por ende la falta de agua causa las pérdidas de cosecha cuando se secan las plantaciones y la muerte de los animales a falta de pastos y agua. Ambos casos de anomalías naturales, alteran el proceso cultural en el tiempo y en el espacio, para controlar estos fenómenos naturales, los hombres andinos diseñaron estrategias y desarrollaron técnicas para el almacenamiento de agua, los reservorios quchakuna.

Las anomalías naturales como la concentración de nieve o lluvia para la diferencia puede traer consecuencias, el primero acumula hielo, el segundo no acumula produce inundaciones. En el mundo andino antiguo, este tipo de anomalías manejaron con equidad y conocimiento de su entorno, es así que construyeron los diques para almacenar agua y utilizar en meses de secanos de producción de alimentos y consumo humano. En el caso andino, la relación con la madre tierra es la base de existencia y la forma adecuada de mantener modos de vida construidos en perfecta simbiosis con leyes de la naturaleza, es decir, previeron mediante las obras hidráulicas la seguridad del agua para los humanos y para la biodiversidad. El agua es el recurso esencial y de ella depende la vida humana, como la existencia de incontables organismos que van desde microscópicos a los anfibios, aves y animales terrestres de todo tipo.

A lo largo de milenios el ser humano ha tenido una influencia creciente sobre los recursos de agua que forman parte de actividades agropecuarias y de consumo humano. En los Andes, las lagunas y los ríos han atraído a los humanos que trastornaron con construcciones de represas, regadíos y otras prácticas agrícolas desde el advenimiento de la civilización. Las tecnologías desarrolladas también manifiestan una necesidad a la escasez, debido al cambio climático, una práctica de remediar la falta de agua, a este nivel responde el registro de las obras hidráulicas, en la jurisdicción del distrito de Huaccana - Apurímac. 


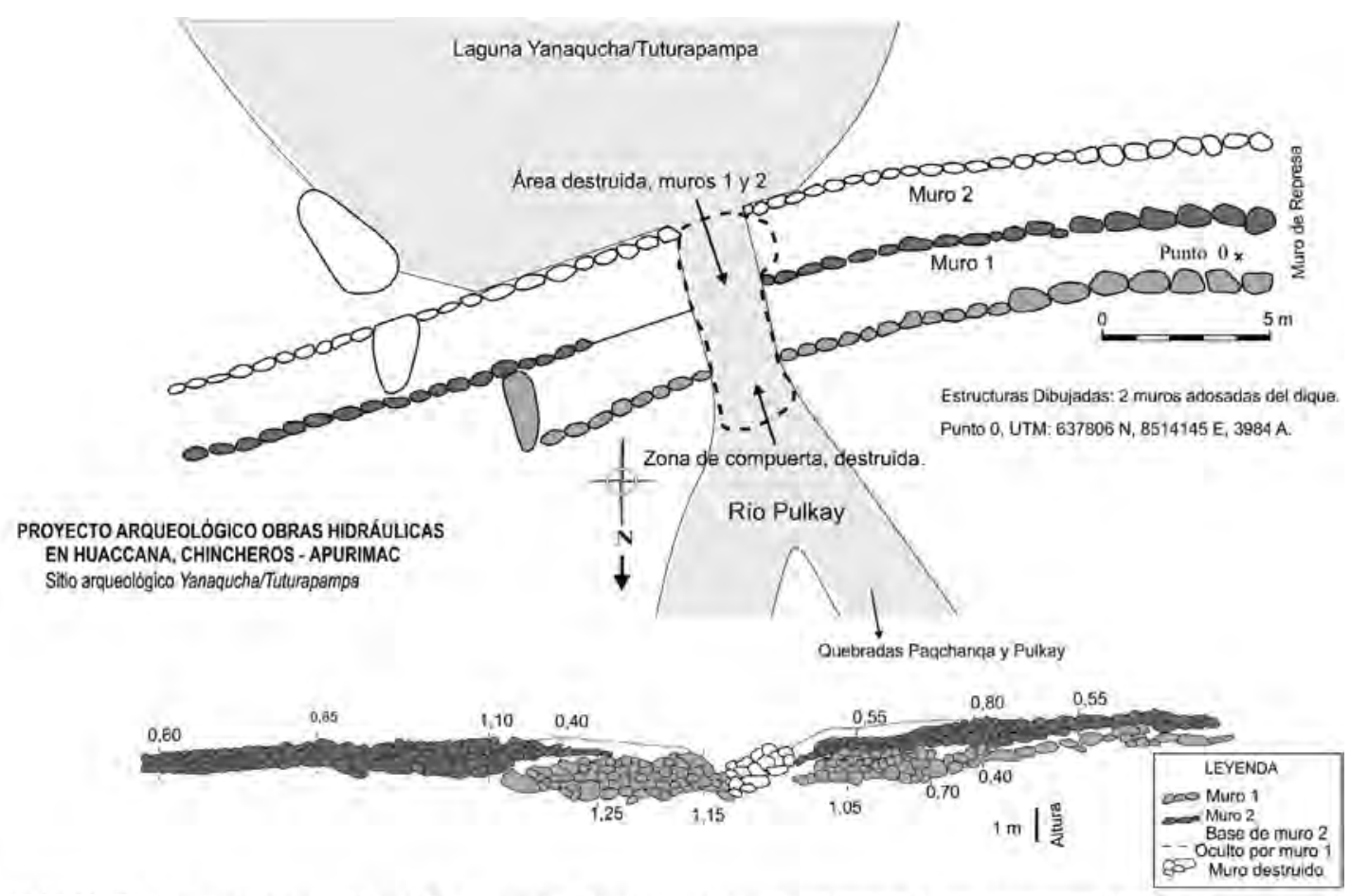

Figura 7: Represa Inka en la Laguna de Yanaqucha/Tuturapampa, véase doble muro el dique y compuerta destruido,

Las tres lagunas de Huaccana, manifiesta la presencia de las obras de ingeniería que son el almacenamiento de agua de la época Inka. Ann Kendall hizo un registro amplio para la zona sur de Ayacucho, así como en Apurímac y Cusco, el experimento de la incorporación mediante una restauración a la actividad agrícola actual de reservorios quchakuna y canales yarqakuna de época prehispánica, Kendall (1992), Kendall et al. (2002 y 2009). Es una necesidad rescatar las tecnologías andinas antiguas para contrarrestar el cambio climático. Rescatar y valorar tecnologías de ingeniería hidráulica es una alternativa, lo cual debería ser con materia prima del lugar y con técnicas locales, la cosecha de agua, la distribución adecuada, equitativa y controlada con organización social del sistema andino yakukamayuq el que distribuye agua, el protagonista en yarqa aspy con procedimiento ritual de la cosmovisión andina, Machaca (2009), Mendoza y Campos (s/f) y Robles (2010).

Los graves problemas ambientales en los Andes básicamente se deben a la destrucción sistemática de la naturaleza. En respuesta a anomalías naturales en el Perú antiguo se construyeron los andenes, útiles para producir y evitar la erosión de suelos, ayudan a regular los flujos de agua y forman parte de una agricultura que contribuye el sostenimiento de la biodiversidad, a embellecer el paisaje, a utilizar energías renovables y no contaminantes, lastimosamente demandan mucha inversión de trabajo y no son rentables. De modo que, hoy en día existe un abandono constante de la tecnología tradicional, se formula la hipótesis que las obras hidráulicas el tratamiento de lagunas en Huaccana, responde a la administración ordenada en la época Inka; sin embargo la sequía de los últimos tiempos agravada por el calentamiento de la tierra 'cambio climático' son dificultades ante la mirada y contemplación del hombre, sin objeción alguna, Araujo (2008) y Llosa et al. (2009). Disfrutando de tecnologías antiguas, en la misma magnitud se puede rescatar con la ejecución tradicional, la eliminación de los sedimentos colmatados, en convenio con las comunidades que deben asumir y garantizar su preservación, PRA- 
TEC (2009). Atestiguar el almacenamiento de agua para mitigar efectos de sequía, el comportamiento climático inestable, podría ser la respuesta a las anomalías naturales, Llosa et al. (2009) ejemplo como la cosecha de agua en el pueblo andino de Quispillacta, Machaca (2009).

\section{Palabras Finales}

Disponiendo los datos arqueológicos podemos reconstruir el clima en el pasado de esta región andina e indagar los efectos del cambio climático, las alteraciones naturales en sociedades tardías del Perú antiguo. Nuestra investigación nos ha llevado a reconocer posibles cambios que afectaron de manera frecuente la llegada de "El Niño" a la región de la sierra de los Andes centrales, al parecer se asocian con importantes transformaciones en la organización de las sociedades del antiguo Perú; a esto responde el registro de los restos de obras hidráulicas en las tres lagunas de Huaccana, son acciones para asegurar el agua, en relación a la presencia de anomalías naturales por ejemplo el efecto de El Niño, a esto se suma las heladas, granizadas y otros.

Los fenómenos naturales causaron fuertes cambios en las sociedades prehispánicas y en algunos casos peligraron su existencia, quizás les obligaron las migraciones forzadas. El fenómeno de El Niño, ocasionó un fuerte impacto en la cosmología andina, son eventos que en los vocabularios de quechua runa simi y aimara se relatan en los mitos. El hombre andino asoció estos eventos excepcionales a actuaciones divinas que marcaban su historia, es así, los mitos y las leyendas también ofrecen valiosa información sobre fenómenos naturales ocurridos, enmarcados en la imaginación que son eventos de significado especial.

La importancia de obras hidráulicas en las lagunas de Huaccana es el conocimiento de técnicas de construcción y la utilización de la materia prima del lugar. La historia de la tecnología andina busca trascender estos principios, poniendo en tela de juicio el fetichismo, una mirada más justa y objetiva, de la tecnología como un hecho social total, a este nivel de organización expresa las obras de ingeniería las represas de las tres lagunas en cadena de Huaccana. La recuperación de tecnologías andinas el sistema de almacenamiento y distribución de agua, mediante canales y reservorios, son los elementos materiales que necesariamente superponen saberes, mentalidades y costumbres del Perú antiguo, en la micro cuenca de Pulkay y la quebrada Paqchanqa, existe evidencias de canales y reservorios más pequeños asociados a las represas de las lagunas.

Recuperar las tecnologías andinas no significa volver al pasado, implica fortalecer la identidad cultural y la capacidad organizacional de los pueblos andinos. No se trata de retornar a un pasado, es buscar y repensar los vínculos entre el desarrollo y las tecnologías para aprender de los errores y aciertos del Perú prehispánico, que manejaron con responsabilidad la tutela de recursos naturales y la escasez de agua tanto para la agricultura y uso doméstico. Es así, las anomalías naturales que afectaba a la población, resolvieron con la observación y adaptación de la nieve acumulada en las montañas y el deshielo que proporciona agua necesaria para la agricultura. Entonces, el conocimiento ambiental, implica la capacidad de enlazar prácticas y saberes tradicionales para enfrentar el cambio climático en los Andes.

No podemos entender el problema del cambio climático ni el retroceso glaciar sin pensar en la historia, sin comprender como la remediaron los antiguos peruanos y se adaptaron a nuevas anomalías naturales. Es ineludible pensar que los problemas medioambientales son dificultades en los Andes centrales, las evidencias arqueológicas de Huaccana, son obras hidráulicas que debieron resolver la sequía y el aridez de meses secanos, todo a través de la sociedad y no solo con la ciencia y la ingeniería, detrás de las obras hay una organización social en el trabajo y fiestas asociadas a grandes banquetes, 
ejemplo las celebraciones de yarqa aspy. Necesitamos una visión mucha más amplia, del proceso prehispánico para ver como controlaron con las obras hidráulicas las lagunas en el área de estudio, para resolver en tiempos secanos la escasez de agua para resolver la aridez.

En resumen, hoy el agua es tratado como objeto, como una cosa, intercambiable, definible por una fórmula química, con lo que pierde toda su esencia espiritual y tradicional que práctica el mundo andino. Esta confrontación de conceptos, lenguajes, valores, actitudes frente a la naturaleza y sus bienes, produce múltiples asociaciones con la interculturalidad, se produce los conflictos entre los naturales y el Estado que explotan los recursos naturales, entre los calendarios nacionales y los cientos de costumbres, fiestas, tradiciones así como el uso del lenguaje runa simi, expresiones culturales ignorada que produce malestar y resentimiento.

\section{Agradecimientos}

Nuestro reconocimiento a las autoridades, al señor alcalde Hugo Najarro Rojas y a los señores (as) regidores(as) del distrito de Huaccana; al señor alcalde Israel Baldeón Ccellccascca y a los señores(as) regidores(as) del distrito de Ongoy. Expresamos nuestra gratitud a los señores presidentes de la Junta Directiva Comunal de ambos distritos, quienes nos han apoyado, trasmitiéndonos su sabiduría acerca de las obras hidráulicas de la época prehispánica en las lagunas naturales. Mi gratitud a Ernesto Valdez y Antonio Pizarro, integrantes del proyecto "Quchakuna: obra hidráulica de época prehispánica en Huaccana, Chincheros - Apurímac" quienes me acompañaron en el trabajo de campo, que al inicio formaban parte del equipo de investigación.

\section{BibliografíA}

ALTAMIRANO, Teófilo

2014 Refugiados ambientales. Cambio climático y migración forzada. PUCP - Fondo Editorial. Lima.

ANTÚNEZ DE MAYOLO, Santiago

1986 Hidráulica costera prehispánica. Allpanchis: Antigüedad y actualidad del riego en los Andes. №. 27, Año XVIII., pp.11-38. Editor Instituto de Pastoral Andina, Cusco - Perú.

ARAUJO, Hilda

2008 Estrategias de las comunidades campesinas altoandinas frente al cambio climático. En Los Andes y las Poblaciones Altoandinas en la Agenda de la Regionalización y la Descentralización. Araujo Camacho, Hilda G., Editora Tomo I. (pp. 169-200). 1ra. Edición, CONCYTEC. Lima.

ATLAS DEL PERÚ

2009 (Geográfico, económico y cultural) - Ayacucho-Apurímac. Tomo 7. Suplemento de El Comercio. Lima.

ÁVILA, Francisco de

1966 [1598] Dioses y Hombres de Huarochiri. Museo Nacional de Historia e Instituto de Estudios Peruanos. Lima.

BERROCAL A. Marcelina

2014 Lagunas: Espacios sagrados y sistemas hidráulicos prehispánicos. Alteritas Revista de Estudios Socioculturales Andino Amazónicos, Año 3, N 3; pp.: 121-148. Universidad Nacional de San Cristóbal de Huamanga. Ayacucho. 


\section{CÁCERES CH., Efraín}

2002 El juicio del agua "Unu Huishu": simbolismo y significado ecológico del agua en mitos andinos. "El milagro de la laguna salada" de Musuq Llaqta. Colección Hombre y Ambiente N 65-66. Ediciones Abya Yala. Ecuador.

CARRIÓN CACHOT, Rebeca,

2005 El culto al agua en el antiguo Perú [1955]. Editorial Instituto Nacional de Cultura. Lima.

CRDCA Comité Regional de Defensa Civil Apurímac

2005-2015 Plan Regional de Prevención y Atención de Desastres - Apurímac. Apurímac.

DE LA TORRE, Carlos y Manuel Burga

1989 Andenes y camellones en el Perú Andino. Historia, presente y futuro. (CONCYTEC). Lima.

DENEVAN, W. M.

1980 Tipología de configuraciones agrícolas prehispánicas. América Indígena, Año XL, Vol. XL, № 4. Instituto Indigenista Interamericano. México.

FARRINGTON, Ian S.

1978 Irrigación prehispánica y establecimiento en la costa norte del Perú. Tecnología Andina, pp. 117-128. 1ra. Edición. IEP; Perú.

GELLES, Paul

1986 Sociedades Hidráulicas en los Andes: Algunas perspectivas desde Huarochirí. Allpanchis: Antigüedad y actualidad del riego en los Andes. № 27, Año XVIII, (pp.99-148). Editor Instituto de Pastoral Andina. Cusco.

GONZÁLEZ HOLGUÍN, Diego

1989 [1608] Vocabulario de la lengua general de todo el Perv llamada qquichua o del Inca. Universidad Nacional Mayor de San Marcos. Lima.

GUAMAN POMA DE AYALA, Felipe

1936 [1615] Nueva Coronica y Buen Gobierno. Institut d'Ethnologie, Paris. Francia.

HERRERA W., Alexander

2012 La recuperación de tecnologías indígenas: Arqueología, tecnología y desarrollo en los Andes. Editorial CLACSO, Universidad de los Andes, IEP. Lima.

2013 Arqueología y Desarrollo en América del Sur. De la práctica a la teoría. Compilador Alexander Herrera W. Ediciones Uniandes y IEP Instituto de Estudios Peruanos. Lima.

HUERTAS, Lorenzo

2001 Diluvios andinos a través de las fuentes documentales. Fondo Editorial Pontificia Universidad Católica del Perú. Lima.

2003 Vice. Ecología, historia y tradición. Municipalidad de Vice. Lima.

2006 Los oráculos en la historia andina. Colección Realidad Nacional, Editorial Universitaria de la Universidad Ricardo Palma. Lima. 


\section{KENDALL, A.}

1992 Infraestructura agrícola e hidráulica prehispánica presente y futuro. Arqueología y Desarrollo Rural. Proyecto Cusichaca Trust, Asociación Gráfica Educativa. Cusco.

KENDALL, A. y RODRÍGUEZ A.

2002 Las cochas andinas: una solución para mitigar el riesgo agropecuario y doméstico en la sierra del Perú. Antropología de sistemas tradicionales de manejo de agua. Vol. III, (pp. 241-255). Editor J. Palerm, Colegio de Postgraduados, Universidad Autónoma de Chapingo. México.

2009 Desarrollo y Perspectivas de los Sistemas de Andenerías en los Andes Centrales del Perú. Traducción Rosalía Ávalos de Matos. Editores Instituto Francés de Estudios Andinos-IFEA, Centro de Estudios Regionales Andinos Bartolomé de Las Casas-CBC. 1ra. Edición. Cusco.

LANE, Kevin

$2007 \mathrm{~m}$ Manejo del agua en la quebrada Las Arcas. Informed preliminary, University of Manchester. EE. UU.

2013 Entre el agua y la pared: patrimonio, desarrollo, campesinos y arqueólogos en la cordillera Negra, Perú. Arqueología y desarrollo en América del Sur. De la práctica a la teoría. Compilador Alexander Herrera W. Ediciones Uniandes y IEP Instituto de Estudios Peruanos. Lima. pp.: 97-118.

\section{LLOSA LARRABURE, Jaime; PAJARES GARAY, Erick y Oscar TORO QUINTO}

2009 Introducción. Cambio climático, crisis del agua y adaptación en las montañas andinas. Reflexión, denuncia y propuesta desde los Andes. Editores Jaime Llosa Larrabure, Erick Pajares Garay y Oscar Toro Quinto. Centro de Estudios y Promoción del Desarrollo DESCO y RAP Red Ambiental Peruana. Lima. pp.: 13-22.

MACHACA, Magdalena

2009 Cosecha y siembra del agua como alma del paisaje. Cambio Climático y Sabiduría Andino Amazónica - Perú. Prácticas, percepciones y adaptaciones indígenas, pp. 108 - 114. Proyecto Andino de Tecnologías Campesinas (PRATEC). Lima.

MATOS, Ramiro

1980 Al Agricultura prehispánica en las punas de Junín. Allpanchis: La Agricultura Andina. Tomo II., pp.91-108. Volumen XIV, $N^{\circ}$. 15. Editor Instituto de Pastoral Andina. Cusco.

MENDOZA B., Alfredo y Nancy CAMPOS P.

s/f Crianza del agua y tradiciones ambientales en Andahuaylas: prácticas campesinas para contrarrestar el cambio climático y fortalecer la soberanía alimentaria. Andahuaylas.

ORTLOFF, C.R.

1985 La ingeniería hidráulica Chimú. La Tecnología en el Mundo Andino, (pp. 91-134). 2da. Edición. Universidad Nacional Autónoma de México. México.

PALERM, A.

1973 Obras hidráulicas prehispánicas en el sistema lacustre del Valle de México. Instituto Nacional de Antropología e Historia, pp. 19-44. INAH. México. 
PARDO, Elena

2009 Vivencia de la Religiosidad Andina y la Crianza del Cambio Climático. Cambio Climático y Sabiduría Andino Amazónica-Perú. Prácticas, percepciones y adaptaciones indígenas, pp. 87-92. Proyecto Andino de Tecnologías Campesinas (PRATEC). Lima.

PARSONS, R. Jerrey y Norbert P. PSUTY

1985 Chacras hundidas y subsistencia prehispánica en la costa del Perú. La tecnología en el Mundo Andino. 2da Edición, pp.51-90. Universidad Nacional Autónoma de México. México.

PRATEC

2009 Cambio Climático y Sabiduría Andino Amazónica - Perú. Prácticas, percepciones y adaptaciones indígenas. Proyecto Andino de Tecnologías Campesinas (PRATEC). Lima.

RAVINES, Rogger

1978 Tecnología andina. IEP. Lima.

RAVINES, R. y SOLAR LA CRUZ, F.

1980 Hidráulica agrícola prehispánica. Allpanchis Phuturinqa La Agricultura Andina, Tomo II., pp. 69-82. Volumen XIV. N ${ }^{\circ}$; Instituto Pastoral Andino. Cusco.

REGAL MATIENZO, Alberto

2005 [1970] Los trabajos hidráulicos del inca en el antiguo Perú. Instituto Nacional de Cultura. Lima.

ROBLES MENDOZA, Román

2010 Sistemas de riego y ritualidad andina en el valle de Colca. Revista Española de Antropología Americana, pp. 197-217.

ROSTWOROWSKI, María

2001 Leyendas y mitos sobre el fenómeno el Niño. El Fenómeno "El Niño" en la costa norte del Perú a través de la historia. Perú-Ecuador: un espacio compartido. Primera y segunda Jornadas de Historia, Universidad de Piura, 152 p. Piura.

SMITH, Clifford T., William M. DENEVAN y Patrick HAMILTON

1985 Antiguos campos de camellones en la región del Lago Titicaca. La Tecnología en el Mundo Andino, (pp. 25-50), 2da. Edición. Universidad Nacional Autónoma de México. México.

TREACY, J. M.

1994 Las chacras de Coporaque. Andenería y riego en el Valle del Colca. Editor Instituto de Estudios Peruanos (IEP). Lima.

URIOSTE, Jorge L.

1980 [1615] Estudio analítico del Quechua en la Nueva Crónica. El Primer Nueva Coronica y buen gobierno. John V. Murra y Rolena Adorno, editores, volumen 1, (pp. XX-XXXI). Ediciones Siglo Veintiuno. México.

Vivanco, Cirilo

2010 ms Prospección arqueológica en Huaccana y Ongoy, Chincheros - Apurímac. Informe final elaborado y presentado a la Dirección de Arqueología del Ministerio de Cultura. Lima. 
2011ms Investigaciones Arqueológicas en Huaccana y Ongoy, Chincheros - Apurímac. Informe presentado a la oficina de Investigaciones, Universidad Nacional de San Cristóbal de Huamanga. Ayacucho.

ZEGARRA, Jorge M.

1978 Irrigación y técnicas de riego en el Perú precolombino. Tecnología Andina, 1ra. Edición, (pp. 107-116). Editor IEP. Lima.

ZEGARRA, Carlos B.

2011 Fenómenos naturales y su interpretación en la etapa prehispánica. Revista Mercurio Peruanos $N^{\circ}$ 524, pp.: 101-117. Editorial Universidad de Piura. Piura. 
